\title{
ON THE RECURSIVE STRUCTURE OF BRANSON'S $Q$-CURVATURES
}

\author{
ANDREAS JUHL
}

\begin{abstract}
We prove universal recursive formulas for Branson's $Q$-curvatures in terms of respective lower-order $Q$-curvatures, lower-order GJMS-operators and renormalized volume coefficients.
\end{abstract}

\section{Introduction and formulation of the main result}

On any Riemannian manifold $(M, g)$ of even dimension $n$, there is a finite sequence $P_{2}(g), P_{4}(g), \ldots, P_{n}(g)$ of differential operators of the form ${ }^{1}$

$$
\Delta_{g}^{N}+\text { lower-order terms, }
$$

which are conformally covariant in the sense that

$$
e^{\left(\frac{n}{2}+N\right) \varphi} P_{2 N}\left(e^{2 \varphi} g\right)(u)=P_{2 N}(g)\left(e^{\left(\frac{n}{2}-N\right) \varphi} u\right), \quad u \in C^{\infty}(M)
$$

for all $\varphi \in C^{\infty}(M)$. Similarly, on manifolds of odd dimension there is an infinite sequence $P_{2}(g), P_{4}(g), \ldots$ of such operators satisfying (1.2). These operators were constructed in [7] from the powers of the Laplacian of an associated ambient metric in the sense of Fefferman and Graham. The lower-order terms of the operators $P_{2 N}(g)$ are determined by the curvature of $g$ and its covariant derivatives. In the following, they will be referred to as the GJMS-operators.

The zeroth order terms of the GJMS-operators lead to the notion of Branson's $Q$-curvatures (see [2]). In fact, Branson proved that, for $2 N<n$, it is natural to write the zeroth order term of $P_{2 N}$ in the form

$$
P_{2 N}(g)(1)=(-1)^{N}\left(\frac{n}{2}-N\right) Q_{2 N}(g)
$$

with a scalar Riemannian curvature invariant $Q_{2 N}(g) \in C^{\infty}(M)$ of order $2 N$. For even $n$, the critical GJMS-operator $P_{n}$ has vanishing constant term and (1.3) cannot be used to define an analogous quantity $Q_{n}$. However, $Q_{n}$ can be defined through $Q_{2 N}$ for $2 N<n$ by a continuation in the dimension. The quantities $Q_{2 N}$ are known as Branson's $Q$-curvatures. For even $n, Q_{n}$ will be called the critical $Q$-curvature.

The following two special cases are well-known. We have

$$
Q_{2}=\frac{\text { scal }}{2(n-1)},
$$

Received by the editors October 5, 2013.

2010 Mathematics Subject Classification. Primary 53B20 53A30; Secondary 58J50.

${ }^{1}$ We use the convention that $-\Delta \geq 0$. 
and

$$
Q_{4}=\frac{n}{2} \mathrm{~J}^{2}-2|\mathrm{P}|^{2}-\Delta \mathrm{J}, n \geq 3
$$

where

$$
\mathrm{J}=\frac{\mathrm{scal}}{2(n-1)} \quad \text { and } \quad \mathrm{P}=\frac{1}{n-2}(\text { Ric }-\mathrm{J} g)
$$

$\mathrm{P}$ is the Schouten tensor. Note that $\mathrm{J}=\operatorname{tr}(\mathrm{P})$. The quantities $Q_{2}$ and $Q_{4}$ appear in the corresponding Yamabe and Paneitz operators

$$
P_{2}=\Delta-\left(\frac{n}{2}-1\right) Q_{2}
$$

and

$$
P_{4}=\Delta^{2}+\delta((n-2) \mathrm{J}-4 \mathrm{P}) d+\left(\frac{n}{2}-2\right) Q_{4} .
$$

The main purpose of this paper is to prove recursive formulas for all higher-order $Q$-curvatures.

In order to formulate the main result, we need some more notation. First, a sequence $I=\left(I_{1}, \ldots, I_{r}\right)$ of integers $I_{j} \geq 1$ will be regarded as a composition of the sum $|I|=I_{1}+I_{2}+\cdots+I_{r}$, where two representations which contain the same summands but differ in the order of the summands are regarded as different. $|I|$ will be called the size of $I$. For $I=\left(I_{1}, \ldots, I_{r}\right)$, we set

$$
P_{2 I}=P_{2 I_{1}} \circ \cdots \circ P_{2 I_{r}} .
$$

We define the multiplicity $m_{I}$ of the composition $I$ by

$$
m_{I}=-(-1)^{r}|I| !(|I|-1) ! \prod_{j=1}^{r} \frac{1}{I_{j} !\left(I_{j}-1\right) !} \prod_{j=1}^{r-1} \frac{1}{I_{j}+I_{j+1}} .
$$

Here, an empty product has to be interpreted as 1 . Note that $m_{(N)}=1$ for all $N \geq 1$ and

$$
\sum_{|I|=N} m_{I}=0
$$

(see Lemma 2.1 in [10]). In these terms, we introduce the generating function

$$
\mathcal{G}(r)=1+\sum_{N \geq 1}\left(\sum_{a+|J|=N} m_{(J, a)}(-1)^{a} P_{2 J}\left(Q_{2 a}\right)\right) \frac{r^{N}}{N !(N-1) !}
$$

Here, for even $n$, the sum on the right-hand side is to be understood as a finite sum over $1 \leq N \leq n$.

A second ingredient of our formula for $Q$-curvatures comes from Poincaré-Einstein metrics. Let $n$ be even. For a given metric $g$ on the manifold $M$ of dimension $n$, let

$$
g_{+}=r^{-2}\left(d r^{2}+g_{r}\right)
$$

with

$$
g_{r}=g+r^{2} g_{(2)}+\cdots+r^{n-2} g_{(n-2)}+r^{n}\left(g_{(n)}+\log r \bar{g}_{(n)}\right)+\cdots
$$


be a metric on $X=(0, \varepsilon) \times M$ so that the tensor $\operatorname{Ric}\left(g_{+}\right)+n g_{+}$satisfies the Einstein condition

$$
\operatorname{Ric}\left(g_{+}\right)+n g_{+}=O\left(r^{n-2}\right)
$$

together with a certain vanishing trace condition. These conditions uniquely determine the coefficients $g_{(2)}, \ldots, g_{(n-2)}$ and the quantity $\operatorname{tr}_{g}\left(g_{(n)}\right)$. They are given as polynomial formulas in terms of $g$, its inverse, the curvature tensor of $g$, and its covariant derivatives. A metric $g_{+}$with these properties is called a Poincaré-Einstein metric with conformal infinity $[g]$. Similarly, for odd $n$, the Einstein condition determines all coefficients in the formal power series

$$
g_{r}=g+r^{2} g_{(2)}+r^{4} g_{(4)}+\cdots
$$

with only even powers of $r$. For full details see [3]. The volume form of $g_{+}$can be written as

$$
\operatorname{vol}\left(g_{+}\right)=r^{-n-1} v(r) d r \operatorname{vol}(g)
$$

where

$$
v(r)=\operatorname{vol}\left(g_{r}\right) / \operatorname{vol}(g) \in C^{\infty}(M) .
$$

The coefficients in the Taylor series

$$
v(r)=v_{0}+v_{2} r^{2}+v_{4} r^{4}+\cdots
$$

are known as the renormalized volume coefficients [5,6] or holographic coefficients [11]. The coefficient $v_{2 j} \in C^{\infty}(M)$ is given by a local formula which involves at most $2 j$ derivatives of the metric.

The following theorem is the main result of the present paper.

Theorem 1.1. On any Riemannian manifold $M$ of dimension $n \geq 3$, we have

$$
\mathcal{G}\left(\frac{r^{2}}{4}\right)=\sqrt{v(r)} .
$$

Some comments are in order. The relation (1.10) is to be understood as an identity of formal power series in $r$. Moreover, for even $n$, it is to be understood as an identity of finite power series terminating at $r^{n}$. The Taylor coefficients of

$$
w(r)=\sqrt{v(r)}=1+w_{2} r^{2}+w_{4} r^{4}+\cdots
$$

can be expressed in terms of the holographic coefficients $v_{2}, v_{4}, \ldots$ In particular, we have

$$
\begin{aligned}
2 w_{2} & =v_{2}, \\
8 w_{4} & =4 v_{4}-v_{2}^{2}, \\
16 w_{6} & =8 v_{6}-4 v_{4} v_{2}+v_{2}^{3}, \\
128 w_{8} & =64 v_{8}-32 v_{6} v_{2}-16 v_{4}^{2}+24 v_{2}^{2} v_{4}-5 v_{2}^{4} .
\end{aligned}
$$

Note that

$$
-2 v_{2}=\mathrm{J} \quad \text { and } \quad 8 v_{4}=\mathrm{J}^{2}-|\mathrm{P}|^{2} .
$$


In [6], Graham gave an algorithm for deriving formulas for holographic coefficients in terms of the (covariant derivatives of the curvature of the) metric, and displayed explicit formulas for $v_{6}$ and $v_{8}$. In particular,

$$
-48 v_{6}=6 \operatorname{tr}\left(\wedge^{3} \mathrm{P}\right)-2\left(\Omega^{(1)}, \mathrm{P}\right)
$$

where $\Omega^{(1)}$ denotes Graham's first extended obstruction tensor. ${ }^{2}$ We refer to [11] for the details of such calculations. Note also that for locally conformally flat metrics,

$$
(-2)^{N} v_{2 N}=\operatorname{tr}\left(\wedge^{N} \mathrm{P}\right)
$$

Theorem 1.1 provides recursive formulas for $Q_{2 N}$ in the following way. For any $N \geq 1$ (so that $2 N \leq n$ if $n$ is even), (1.10) states that

$$
\sum_{a+|J|=N} m_{(J, a)}(-1)^{a} P_{2 J}\left(Q_{2 a}\right)=2^{2 N} N !(N-1) ! w_{2 N} .
$$

One of the $2^{N-1}$ items in the sum on the left-hand side of $(1.13)$ is $(-1)^{N} Q_{2 N}$. All other items are defined in terms of lower-order GJMS-operators acting on lower-order $Q$-curvatures.

The relation (1.13) can be regarded as a formula for the difference

$$
Q_{2 N}-(-1)^{N} 2^{2 N-1} N !(N-1) ! v_{2 N} .
$$

An alternative formula for the same difference is given by the holographic formula

$$
2 N c_{N} Q_{2 N}=\sum_{j=0}^{N-1}(N-j) \mathcal{T}_{2 j}^{*}\left(\frac{n}{2}-N\right)\left(v_{2 N-2 j}\right)
$$

for the notation see Section 3. In the critical case $2 N=n$, the latter formula was proved in [8]. For the general case we refer to [12].

The identities (1.10) are valid in all dimensions. This feature will be referred to as universality.

For the convenience of the reader, we display the explicit formulas for the four lowest order $Q$-curvatures. We find

$$
\begin{aligned}
& Q_{2}=-4 w_{2}, \\
& Q_{4}=-P_{2}\left(Q_{2}\right)+2^{4} 2 ! w_{4} \\
& Q_{6}=-2 P_{2}\left(Q_{4}\right)+2 P_{4}\left(Q_{2}\right)-3 P_{2}^{2}\left(Q_{2}\right)-2^{6} 3 ! 2 ! w_{6}
\end{aligned}
$$

and

$$
\begin{aligned}
Q_{8}=-3 & P_{2}\left(Q_{6}\right)-3 P_{6}\left(Q_{2}\right)+9 P_{4}\left(Q_{4}\right) \\
& +8 P_{2} P_{4}\left(Q_{2}\right)-12 P_{2}^{2}\left(Q_{4}\right)+12 P_{4} P_{2}\left(Q_{2}\right)-18 P_{2}^{3}\left(Q_{2}\right)+2^{8} 4 ! 3 ! w_{8}
\end{aligned}
$$

\footnotetext{
${ }^{2}$ In more familiar terms, $\Omega^{(1)}$ equals $-\mathcal{B} /(n-4)$, where $\mathcal{B}$ is the Bach tensor in dimension $n$.
} 
By $P_{2}=\Delta-\left(\frac{n}{2}-1\right) \mathrm{J}$ and (1.11), the formula for $Q_{4}$ is easily seen to be equivalent to (1.4). Similarly, combining formula (1.14) with recursive formulas for $P_{4}$ and $P_{6}$ yields more explicit presentations of $Q_{8}$. For the details concerning such consequences we refer to [13].

For round spheres $\mathbb{S}^{n}$, Theorem 1.1 can be proved by direct summation of the left-hand side (see [10]).

Theorem 1.1 was used in [14] to prove recursive formulas for the full GJMSoperators. The proofs rest on the theory of residue families as developed in [11]. In [4], it was shown how these formulas also can be derived from the perspective of the original ambient metric construction. This approach also yields an alternative proof of Theorem 1.1. The latter arguments naturally extend to the pseudo-Riemannian setting. For a direct elementary proof of Theorem 1.1 on the pseudo-Riemannian $\mathbb{S}^{p, q}$ we refer to [15].

The paper is organized as follows. In Section 2, we establish explicit formulas for a sequence of recursively defined operator-valued polynomials $\pi_{2 N}(\lambda)$. These are closely related to the $Q$-curvature polynomials $Q_{2 N}^{\text {res }}(\lambda)$ introduced in [11]. In Section 3, we recall this concept and show that the relation implies Theorem 1.1, when combined with a result of [13].

\section{The polynomials $\pi_{2 N}(\lambda)$}

In the present section, we discuss a sequence of operator-valued polynomials which are closely related to the $Q$-curvature polynomials. That relation will be important in Section 3.

We start by defining some higher analogs of the multiplicities $m_{I}$. We set $m_{I}^{(1)}=$ $m_{I}$, and define the rational numbers $m_{I}^{(k)}$ for $k \geq 2$ by the formulas

$$
m_{(a, J)}^{(k)}=\frac{\sum_{j=0}^{k-1} s(N, N-j)|J|^{k-1-j}}{(N-1) \cdots(N-k+1)} m_{(a, J)}^{(1)}
$$

if $a+|J|=N$ and $2 \leq k \leq N-1$, and

$$
m_{(N)}^{(k)}=\frac{s(N, N-k+1)}{(N-1) \cdots(N-k+1)} m_{(N)}^{(1)}
$$

for $2 \leq k \leq N$. Note that (2.2) (for $2 \leq k \leq N-1$ ) can be regarded as the special case $J=(0)$ of $(2.1)$.

Here, $s(n, m)$ are the Stirling numbers of the first kind. These are defined by the generating functions

$$
\sum_{k=0}^{n} s(n, k) x^{k}=x(x-1) \cdots(x-n+1)=b_{n}(x) .
$$

In particular, we have

$$
s(n, 1)=(-1)^{n-1}(n-1) !, \quad s(n, n-1)=-\left(\begin{array}{l}
n \\
2
\end{array}\right) \quad \text { and } \quad s(n, n)=1 .
$$


Note that the definitions show that

$$
m_{(a, J)}^{(2)}=\frac{s(N, N)|J|+s(N, N-1)}{N-1} m_{(a, J)}^{(1)}=\left(\frac{N-a}{N-1}-\frac{N}{2}\right) m_{(a, J)}^{(1)}
$$

if $a+|J|=N$, and

$$
m_{(N)}^{(2)}=\frac{s(N, N-1)}{N-1}=-\frac{N}{2} m_{(N)}^{(1)} .
$$

Finally, we use the operators

$$
\mathbf{C}_{2 N}^{(k)}=\sum_{|I|=N} m_{I}^{(k)} P_{2 I} \quad \text { for } 1 \leq k \leq N-1
$$

and

$$
\mathbf{C}_{2 N}^{(N)}=(-1)^{N-1} P_{2 N}
$$

to define the operator-valued polynomials

$$
\pi_{2 N}(\lambda)=\sum_{k=1}^{N} \mathbf{C}_{2 N}^{(k)} \frac{1}{(N-k) !}\left(\lambda+\frac{n}{2}-N\right)^{N-k}, \quad N \geq 1 .
$$

We display explicit formulas for these polynomials for $N \leq 3$.

Examples 2.1. We have, $\pi_{2}(\lambda)=P_{2}$,

$$
\pi_{4}(\lambda)=\left(P_{4}-P_{2}^{2}\right)\left(\lambda+\frac{n}{2}-2\right)-P_{4}
$$

and

$$
\begin{aligned}
\pi_{6}(\lambda)=( & \left.P_{6}-2 P_{4} P_{2}-2 P_{2} P_{4}+3 P_{2}^{3}\right) \frac{1}{2 !}\left(\lambda+\frac{n}{2}-3\right)^{2} \\
+ & \left(-\frac{3}{2} P_{6}+2 P_{4} P_{2}+P_{2} P_{4}-\frac{3}{2} P_{2}^{3}\right)\left(\lambda+\frac{n}{2}-3\right)+P_{6} .
\end{aligned}
$$

Note that $m_{I}=m_{I^{-1}}$, where $I^{-1}$ is the inverse composition of $I$, i.e., we set $\left(I_{1}, \ldots, I_{r}\right)^{-1}=\left(I_{r}, \ldots, I_{1}\right)$. Since the GJMS-operators are formally self-adjoint (see $[9])$, this fact implies that $\mathbf{C}_{2 N}^{(1)}$ is formally self-adjoint, too.

The main result of the present section consists in the following characterization of the polynomials $\pi_{2 N}(\lambda)$.

Theorem 2.1. For any $N \geq 1$, the polynomial $\pi_{2 N}(\lambda)$ satisfies the $N$ identities

$$
\pi_{2 N}\left(-\frac{n}{2}+2 N-j\right)=(-1)^{j} P_{2 j} \pi_{2 N-2 j}\left(-\frac{n}{2}+2 N-j\right), \quad j=1, \ldots, N-1
$$

and

$$
\pi_{2 N}\left(-\frac{n}{2}+N\right)=(-1)^{N-1} P_{2 N}
$$


Since $\pi_{2 N}(\lambda)$ has degree $N-1$, the factorizations (2.9) and (2.10) uniquely determine this polynomial in terms of the lower-order relatives $\pi_{2}, \ldots, \pi_{2 N-2}$ and the GJMS-operator $P_{2 N}$.

As a preparation of the proof of Theorem 2.1, we establish the following result.

Lemma 2.1. For all $N \geq 2$,

$$
\sum_{2 \leq a+b \leq N} s(N, a+b) x^{a} y^{b}=\frac{y x(x-1) \cdots(x-N+1)-x y(y-1) \cdots(y-N+1)}{x-y}
$$

for $x \neq y$. Moreover,

$$
\sum_{2 \leq a+b \leq N} s(N, a+b) M^{a+b-1}=(-1)^{N-M-1} M !(N-M-1) !
$$

for $M=1, \ldots, N-1$. Here the sums run over all natural numbers $a, b \geq 1$ subject to the condition $2 \leq a+b \leq N$.

Proof. The sum in (2.11) can be written in the form

$$
s(N, N) \sum_{a=1}^{N-1} x^{a} y^{N-a}+s(N, N-1) \sum_{a=1}^{N-2} x^{a} y^{N-a-1}+\cdots+s(N, 2) x y .
$$

Now (2.13) equals

$$
\begin{aligned}
& \sum_{k=2}^{N} s(N, k)\left(y \frac{x^{k}-y^{k}}{x-y}-y^{k}\right) \\
& \quad=\frac{y}{x-y}\left(\sum_{k=2}^{N} s(N, k) x^{k}-\sum_{k=2}^{N} s(N, k) y^{k}\right)-\sum_{k=2}^{N} s(N, k) y^{k} .
\end{aligned}
$$

In view of (2.3), the right-hand side simplifies to

$$
\begin{aligned}
& \frac{y}{x-y}(x(x-1) \cdots(x-N+1)-y(y-1) \cdots(y-N+1)-s(N, 1) x+s(N, 1) y) \\
& -(y(y-1) \cdots(y-N+1)-s(N, 1) y) .
\end{aligned}
$$

Now (2.11) follows from here by a further simplification. Finally, (2.12) follows from (2.11) by taking the limit $x \rightarrow y$ for $y=1, \ldots, N-1$.

We continue with the

Proof of Theorem 2.1. (2.10) is obvious by (2.7). The left-hand side of (2.9) equals

$$
\sum_{k=1}^{N-1} \mathbf{C}_{2 N}^{(k)} \frac{1}{(N-k) !}(N-j)^{N-k}+(-1)^{N-1} P_{2 N}
$$

We prove that all non-trivial contributions to this sum are multiples of the operators

$$
P_{2 j} P_{2 J}, j+|J|=N \text {. }
$$

Moreover, we determine the corresponding weights. Let $J$ be non-trivial. Equation (2.1) shows that the coefficient of $P_{2 j} P_{2 J}$ in

$$
\sum_{k=1}^{N-1} \mathbf{C}_{2 N}^{(k)} \frac{1}{(N-k) !}(N-j)^{N-k}
$$


is given by

$$
\begin{aligned}
& \sum_{k=1}^{N-1}\left(\frac{\sum_{i=0}^{k-1} s(N, N-i)(N-j)^{k-1-i}}{(N-1) \cdots(N-k+1)}\right) \frac{(N-j)^{N-k}}{(N-k) !} m_{(j, J)}^{(1)} \\
& =\frac{1}{(N-1) !} \sum_{k=1}^{N-1} \sum_{i=0}^{k-1} s(N, N-i)(N-j)^{N-1-i} m_{(j, J)}^{(1)} \\
& =\frac{1}{(N-1) !} \sum_{2 \leq i+k \leq N} s(N, i+k)(N-j)^{i+k-1} m_{(j, J)}^{(1)} .
\end{aligned}
$$

Equation (2.12) implies that the latter sum equals

$$
(-1)^{j-1} \frac{(N-j) !(j-1) !}{(N-1) !} m_{(j, J)}^{(1)}=(-1)^{j-1}\left(\begin{array}{c}
N-1 \\
j-1
\end{array}\right)^{-1} m_{(j, J)}^{(1)}
$$

for $1 \leq j \leq N-1$.

Next, (2.3) shows that $P_{2 N}$ contributes to (2.14) with the coefficient

$$
\begin{aligned}
& (-1)^{N-1}+\sum_{k=1}^{N-1} m_{(N)}^{(k)} \frac{1}{(N-k) !}(N-j)^{N-k} \\
& \quad=(-1)^{N-1}+\frac{1}{(N-1) !} \sum_{k=2}^{N} s(N, k)(N-j)^{k-1}=(-1)^{N-1}-\frac{s(N, 1)}{(N-1) !}=0 .
\end{aligned}
$$

In the last step we have used (2.4).

Now let $l \neq j$. The coefficient of

$$
P_{2 l} P_{2 J}, l+|J|=N, 1 \leq l \leq N-1
$$

in $(2.15)$ is given by

$$
\begin{gathered}
\frac{1}{(N-1) !} \sum_{k=1}^{N-1}\left(\sum_{i=0}^{k-1} s(N, N-i)(N-l)^{k-1-i}\right)(N-j)^{N-k} m_{(l, J)}^{(1)} \\
=\frac{1}{(N-1) !}\left(\sum_{2 \leq i+k \leq N} s(N, i+k)(N-l)^{i-1}(N-j)^{k}\right) m_{(l, J)}^{(1)} .
\end{gathered}
$$

Lemma 2.1 implies that this sum vanishes.

Finally, we prove that the weight of $P_{2 j} P_{2 J}$ on the left-hand side of (2.9) coincides with its weight on the right-hand side. For this we write $J=(r, K)$ (with a possibly trivial $K)$. For non-trivial $K$, we have $1 \leq r<N-j$. In this case, the result $(2.16)$ shows that the assertion is equivalent to

$$
\begin{aligned}
& \sum_{k=1}^{N-j-1}\left(\frac{\sum_{i=0}^{k-1} s(N-j, N-j-i)(N-j-r)^{k-1-i}}{(N-j-1) \cdots(N-j-k+1)}\right) \frac{N^{N-j-k}}{(N-j-k) !} \\
& \quad=-m_{(j, r, K)}^{(1)} / m_{(r, K)}^{(1)}\left(\begin{array}{c}
N-1 \\
j-1
\end{array}\right)^{-1} .
\end{aligned}
$$


Using the abbreviation $M=N-j$, the left-hand side of (2.17) equals

$$
\begin{gathered}
\frac{1}{(M-1) !} \sum_{k=1}^{M-1} \sum_{i=0}^{k-1} s(M, M-i)(M-r)^{k-1-i} N^{M-k} \\
=\frac{1}{(M-1) !} \sum_{2 \leq a+b \leq M} s(M, a+b)(M-r)^{a-1} N^{b} .
\end{gathered}
$$

We apply Lemma 2.1 to simplify this sum. We find

$$
\begin{aligned}
& -\frac{1}{(N-j-1) !}\left(\frac{N(M-r) \cdots(-r+1)-(M-r) N(N-1) \cdots(N-M+1)}{(N-j-r)(j+r)}\right) \\
& \quad=\frac{1}{j+r} \frac{N !}{j !(N-j-1) !} .
\end{aligned}
$$

The assertion (2.17) follows by combining this result with

$$
m_{(j, r, K)}^{(1)} / m_{(r, K)}^{(1)}=-\frac{1}{j+r}\left(\begin{array}{c}
N \\
j
\end{array}\right)^{2} \frac{j(N-j)}{N} .
$$

For trivial $K$, i.e., $J=(r)$ and $r=N-j$, the result (2.16) shows that the assertion is equivalent to

$$
\frac{1}{(r-1) !} \sum_{k=1}^{r-1} s(r, r-k+1) N^{r-k}+(-1)^{r-1}=-m_{(j, r)}^{(1)} / m_{(r)}^{(1)}\left(\begin{array}{c}
N-1 \\
j-1
\end{array}\right)^{-1} .
$$

Here, the term $(-1)^{r-1}$ on the left-hand side comes from the contribution of $\mathbf{C}_{2 r}^{(r)}$. By (2.3) and (2.4), the left-hand side equals

$$
\frac{1}{(r-1) ! N}\left[N(N-1) \cdots(N-r+1)+(-1)^{r}(r-1) ! N\right]+(-1)^{r-1}=\frac{(N-1) !}{j !(N-j-1) !} .
$$

On the other hand,

$$
m_{(j, r)}^{(1)} / m_{(r)}^{(1)}=-\frac{1}{N}\left(\begin{array}{c}
N \\
j
\end{array}\right)^{2} \frac{j(N-j)}{N} .
$$

This yields (2.18). The proof is complete.

Remark 2.1. Similar arguments can be used to prove the closed formula

$$
\pi_{2 N}(\lambda)=\frac{1}{(N-1) !} \sum_{|I|=N} \frac{b_{N}\left(\lambda+\frac{n}{2}-N\right)}{\lambda+\frac{n}{2}-2 N+I_{l}} m_{I} P_{2 I}
$$

(see (2.3)). Here $I_{l}$ denotes the most left entry of the composition I. Note that the coefficients in (2.19) are polynomials of degree $N-1$ since

$$
\lambda+\frac{n}{2}-2 N+I_{l}=\left(\lambda+\frac{n}{2}-N\right)-\left(N-I_{l}\right)
$$

and the integers $N-I_{l}$ are zeros of $b_{N}(x)$. We omit the details. 


\section{The recursive structure of $Q$-curvature}

In the present section, we prove Theorem 1.1.

The proof utilizes properties of so-called $Q$-curvature polynomials. The notion of $Q$-curvature polynomials was introduced in [11] (see also [1]). We briefly recall this concept. Assume that $n$ is even. Associated to any Riemannian manifold $(M, g)$ of dimension $n$, there is a finite sequence $Q_{2}^{\text {res }}(g ; \lambda), Q_{4}^{\text {res }}(g ; \lambda), \ldots, Q_{n}^{\text {res }}(g ; \lambda)$ of polynomials of respective degrees $1,2, \ldots, \frac{n}{2}$. These polynomials are defined by the constant terms

$$
Q_{2 N}^{\mathrm{res}}(g ; \lambda)=-(-1)^{N} D_{2 N}^{\mathrm{res}}(g ; \lambda)(1)
$$

of the so-called residue families

$$
D_{2 N}^{\mathrm{res}}(g ; \lambda): C^{\infty}([0, \varepsilon) \times M) \rightarrow C^{\infty}(M) .^{3}
$$

These are families of local operators which are defined in terms of the holographic coefficients $v_{2 j}$ and the coefficients $\mathcal{T}_{2 j}(\lambda)(f)$ in the asymptotic expansion

$$
u \sim \sum_{j \geq 0} r^{\lambda+2 j} \mathcal{T}_{2 j}(\lambda)(f), \quad \mathcal{T}_{0}(\lambda)(f)=f, r \rightarrow 0
$$

of eigenfunctions

$$
-\Delta_{g_{+}} u=\lambda(n-\lambda) u \text {. }
$$

of the Laplace-Beltrami operator for the Poincaré-Einstein metric $g_{+}$corresponding to $g$. The coefficients $\mathcal{T}_{2 j}(g ; \lambda)$ are meromorphic families (in $\lambda$ ) of differential operators on $M$. The residue families are conformally covariant generalizations of the GJMSoperators in the following sense. For any GJMS-operators $P_{2 N}$, the family $D_{2 N}^{\text {res }}(\lambda)$ contains $P_{2 N}$ in the sense that

$$
D_{2 N}^{\mathrm{res}}\left(g ;-\frac{n}{2}+N\right)=P_{2 N}(g) i^{*}
$$

where $i: M \hookrightarrow[0, \varepsilon) \times M$ denotes the embedding $m \mapsto(0, m)$. Moreover, $D_{2 N}^{\text {res }}(g ; \lambda)$ is conformally covariant in the sense that it satisfies the transformation law

$$
e^{-(\lambda-2 N) \varphi} D_{2 N}^{\mathrm{res}}\left(e^{2 \varphi} g ; \lambda\right)=D_{2 N}^{\mathrm{res}}(g ; \lambda) \circ \kappa_{*} \circ\left(\frac{\kappa^{*}(r)}{r}\right)^{\lambda}
$$

for all $\varphi \in C^{\infty}(M)$. Here, $\kappa$ denotes the diffeomorphism which relates the PoincaréEinstein metrics of $g$ and $\hat{g}=e^{2 \varphi} g$, i.e.,

$$
\kappa^{*}\left(r^{-2}\left(d r^{2}+g_{r}\right)\right)=r^{-2}\left(d r^{2}+\hat{g}_{r}\right)
$$

and $\kappa$ restricts to the identity on $r=0$.

\footnotetext{
${ }^{3}$ The name comes from their relation to a certain residue construction (see [11]).
} 
In terms of the families $\mathcal{T}_{2 N}(\lambda)$ and the holographic coefficients, the $Q$-curvature polynomials are defined by

$$
\begin{aligned}
Q_{2 N}^{\mathrm{res}}(h ; \lambda)= & -2^{2 N} N !\left(\left(\lambda+\frac{n}{2}-2 N+1\right) \cdots\left(\lambda+\frac{n}{2}-N\right)\right) \\
& \times\left[\mathcal{T}_{2 N}^{*}(h ; \lambda+n-2 N)\left(v_{0}\right)+\cdots+\mathcal{T}_{0}^{*}(h ; \lambda+n-2 N)\left(v_{2 N}\right)\right] .
\end{aligned}
$$

Here, the overall polynomial factor has the effect to remove poles.

An additional important feature of residue families is that they satisfy a system of factorization identities which generalize (3.2). In fact, we have

$$
D_{2 N}^{\mathrm{res}}\left(g ;-\frac{n}{2}+2 N-j\right)=P_{2 j}(g) D_{2 N-2 j}^{\mathrm{res}}\left(g ;-\frac{n}{2}+2 N-j\right), j=1, \ldots, N .
$$

Here, (3.2) is contained as the special case $j=N$; note that $D_{0}^{\text {res }}(g ; \lambda)=i^{*}$. Now (3.3) implies that

$$
Q_{2 N}^{\mathrm{res}}\left(-\frac{n}{2}+2 N-j\right)=(-1)^{j} P_{2 j} Q_{2 N-2 j}^{\mathrm{res}}\left(-\frac{n}{2}+2 N-j\right), j=1, \ldots, N .
$$

Note that $Q_{0}^{\text {res }}(\lambda)=-1$.

For the details on residue families and $Q$-curvature polynomials we refer to $[1,11$, 14]. In particular, [14] contains a detailed proof of the factorization identities (3.3) (see Theorem 3.1).

We continue with the

Proof of Theorem 1.1. The assertion is equivalent to

$$
\sum_{a+|J|=N} m_{(J, a)}(-1)^{a} P_{2 J}\left(Q_{2 a}\right)=2^{2 N} N !(N-1) ! w_{2 N}, N \geq 1 .
$$

We prove (3.5) by comparing two different evaluations of the leading coefficient of the $Q$-curvature polynomial $Q_{2 N}^{\text {res }}(\lambda)$. First, assume that $n$ is odd. On the one hand, Proposition 4.2 in [13] shows that the coefficient of $\lambda^{N}$ is

$$
-2^{2 N} N ! w_{2 N}
$$

On the other hand, the degree $N$ polynomial $Q_{2 N}^{\text {res }}(\lambda)$ satisfies the identities

$$
Q_{2 N}^{\mathrm{res}}\left(-\frac{n}{2}+2 N-j\right)=(-1)^{j} P_{2 j} Q_{2 N-2 j}^{\mathrm{res}}\left(-\frac{n}{2}+2 N-j\right), j=1, \ldots, N-1
$$

and

$$
Q_{2 N}^{\mathrm{res}}\left(-\frac{n}{2}+N\right)=-\left(\frac{n}{2}-N\right) Q_{2 N}
$$

(see (3.4)). Moreover, an analog of Theorem 1.6.6 in [1] for odd $n$ states the vanishing result ${ }^{4}$

$$
Q_{2 N}^{\text {res }}(0)=0
$$

\footnotetext{
${ }^{4}$ For odd $n$, the proof simplifies since the families $\mathcal{T}_{2 N}(\lambda)$ are regular at $n-2 N$.
} 
These results show that (3.7) and (3.8) are equivalent to the identities

$$
\mathcal{Q}_{2 N}^{\text {res }}\left(-\frac{n}{2}+2 N-j\right)=(-1)^{j} P_{2 j} \mathcal{Q}_{2 N-2 j}^{\text {res }}\left(-\frac{n}{2}+2 N-j\right), \quad j=1, \ldots, N-1
$$

and

$$
\mathcal{Q}_{2 N}^{\text {res }}\left(-\frac{n}{2}+N\right)=Q_{2 N}
$$

for the polynomials

$$
\mathcal{Q}_{2 N}^{\text {res }}(\lambda)=\lambda^{-1} Q_{2 N}^{\text {res }}(\lambda) .
$$

By comparing the relations (3.9) and (3.10) with (2.9) and (2.10), Theorem 2.1 implies that the leading coefficient of $Q_{2 N}^{\text {res }}(\lambda)$ equals

$$
-\frac{1}{(N-1) !} \sum_{|J|+a=N} m_{(J, a)}(-1)^{a} P_{2 J}\left(Q_{2 a}\right) .
$$

Now the equality of (3.6) and (3.12) is equivalent to the asserted identity (3.5). Next, assume that $n$ is even. Then, under the additional assumption $n \geq 4 N$, i.e., $-\frac{n}{2}+2 N \leq 0$, the sets

$$
\left\{-\frac{n}{2}+2 N-j \mid j=1, \ldots, N\right\} \quad \text { and } \quad\{0\}
$$

are disjoint, and the assertion follows by the same arguments as above. Thus, for fixed $N$, we have proved (3.5) in all dimensions $n \geq 4 N$. Now we recall that all quantities in (3.5) are given by universal expressions in terms of the metric, its inverse, the curvature and covariant derivatives thereof with coefficients that are rational functions in $n$ which are regular for $n \geq 2 N$. As a consequence, the relation (3.5) holds true also in the remaining cases $4 N>n \geq 2 N$ (for even $n$ ).

Finally, we observe that Theorem 1.1 is equivalent to

$$
\mathcal{G}^{2}\left(\frac{r^{2}}{4}\right)=v(r)
$$

This formulation naturally expresses the contributions of lower-order holographic coefficients $v_{2 j}(2 j<2 N)$ on the right-hand side of $(1.10)$ in terms of lower-order GJMS-operators acting on lower-order $Q$-curvatures. In fact, comparing coefficients in (3.13) yields the relations

$$
2 \Lambda_{2 N}+\sum_{j=1}^{N-1} \frac{j(N-j)}{N}\left(\begin{array}{c}
N \\
j
\end{array}\right)^{2} \Lambda_{2 j} \Lambda_{2 N-2 j}=2^{2 N} N !(N-1) ! v_{2 N},
$$

where

$$
\Lambda_{2 M} \stackrel{\text { def }}{=} \sum_{|a+| J \mid=M} m_{(J, a)}(-1)^{a} P_{2 J}\left(Q_{2 a}\right) .
$$


In particular, we find

$$
\left(Q_{4}+P_{2}\left(Q_{2}\right)\right)+Q_{2}^{2}=2 ! 2^{3} v_{4}
$$

and

$$
\left(Q_{6}+2 P_{2}\left(Q_{4}\right)-2 P_{4}\left(Q_{2}\right)+3 P_{2}^{2}\left(Q_{2}\right)\right)+6\left(Q_{4}+P_{2}\left(Q_{2}\right)\right) Q_{2}=2 ! 3 ! 2^{5} v_{6}
$$

\section{Acknowledgment}

The work was supported by SFB 647 "Raum-Zeit-Materie" of DFG.

\section{References}

[1] H. Baum and A. Juhl, Conformal differential geometry: Q-curvature and conformal holonomy, Vol. 40 of Oberwolfach Seminars, Birkhuser (2010).

[2] T. Branson, Sharp inequalities, the functional determinant, and the complementary series, Trans. Amer. Math. Soc. 347 (1995), 3671-3742.

[3] C. Fefferman and C.R. Graham, The ambient metric, Vol. 178 of Annals of Mathematics Studies, Princeton University Press (2012).

[4] - Juhl's formulae for GJMS-operators and Q-curvatures, J. Amer. Math. Soc. 26(4) (2013), 1191-1207.

[5] C.R. Graham, Volume and area renormalizations for conformally compact Einstein metrics, Rend. Circ. Mat. Palermo (2) Suppl. 63 (2000) 31-42.

[6] - Extended obstruction tensors and renormalized volume coefficients, Adv. Math. 220(6) (2009), 1956-1985.

[7] C.R. Graham, R. Jenne, L.J. Mason and G.A.J. Sparling, Conformally invariant powers of the Laplacian. I. Existence, J. London Math. Soc. 46(3) (1992), 557-565.

[8] C.R. Graham and A. Juhl, Holographic formula for Q-curvature, Adv. Math. 216(2) (2007), 841-853.

[9] C.R. Graham and M. Zworski, Scattering matrix in conformal geometry, Invent. Math. 152(1) (2003), 89-118.

[10] A. Juhl, On conformally covariant powers of the Laplacian. arXiv: 0905.3992v3.

[11] - Families of conformally covariant differential operators, Q-curvature and holography, Vol. 275 of Progress in Mathematics, Birkhäuser Verlag (2009).

[12] - Holographic formula for Q-curvature. II, Adv. Math. 226(4) (2011), 3409-3425.

[13] - On Branson's Q-curvature of order eight, Conform. Geom. Dyn. 15 (2011), 20-43.

[14] - Explicit formulas for GJMS-operators and Q-curvatures, Geom. Funct. Anal. 23 (2013), $1278-1370$.

[15] A. Juhl and C. Krattenthaler, Summation formulas for GJMS-operators and Q-curvatures on the Möbius sphere, J. Approximation Theory (2014). arXiv: 0910.4840v1.

Humboldt-Universität, Institut für Mathematik, Unter den Linden, D-10099 Berlin, GERMANY

E-mail address: ajuhl@math.hu-berlin.de

University Uppsala, Department of Mathematics, P.O. Box 480, S-75106 Uppsala, SWEDEN

E-mail address: andreasj@math.uu.se 
